\title{
Multiscale porosity an mineral mapping of shales
}

\author{
D. PRET ${ }^{1 *}$
}

${ }^{1}$ IC2MP-HYDRASA, UMR 7285, CNRS/POITIERS Univ., TSA 51106, 86073, Poitiers, France (*correspondence : dimitri.pret@univ-poitiers.fr)

Shales are characterized by multi-scaled pore systems (from micro- to nano-meter scale) associated with variable spatial distributions of mineral grains/particles up to the core scale. Some grains are nearly non-porous (like tectosilicates, carbonates, etc.) whereas the clay matrix is bearing most of the pore network, controlling the storage/sorption areas and migration pathways of fluids/solutes. [1] Pore network is thus strongly dependent on the relative arrangement and content of minerals.

Only a correlative multi-techniques approach is relevant to provide a quantitative balance of the pore size distribution. However, such a balance is not achievable when each of the different analytical methods is applied on randomly selected samples collected from an heterogeneous core. [2] First, a detailled mapping of the mineral distribution is thus needed from the core down to the clay particle scales.

Recent advances of X-ray Energy Dispersive Spectrometers (EDS) coupled with electron/X-ray microscopes allow the fast acquisition of large hyperspectral maps with a good enough counting statistic for a later accurate chemical analysis on each pixel. Applying this approach on $\mu \mathrm{XRF}$, SEM, STEM in SEM or STEM is helpful for probing the mineral/phase distribution at different scales for hierarchical and widely heterogeneous shales. The careful treatment of the quantitative chemical maps provides the identification of the mineral/phase or mixture associated to each pixel [3][4][5]. The mineral/phase map reached then permits a robust quantification of the local phase contents and arrangements at each scale.

When coupled with the quantitative porosity mapping by autoradiography at core scale (resolution $\sim 20 \mu \mathrm{m}$ ) and the pore mapping by mosaics of BackScattered Electron images obtained on SEM at lower scale (resolution $\sim 5 \mathrm{~nm}$ ), a better understanding of the variability of pore network is reached .

[1] Robinet et al. (2013) 48, Wat. Ress. Res., W05554. [2] Matskova et al. (2017) URTEC Proceedings, 2689299. [3] Prêt et al. (2010a) Am. Min., 95, 1379-1388. [4] Prêt et al. (2010b) Am. Min., 95, 1389-1398. [5] Gaboreau et al. (2017) Cement and concrete composites, 83, 360-373. 